\title{
Influence of Dissolved Gas Pressure and Release Angle on the Microbubble Diameter in Single Component Gas-dissolving Method
}

\author{
Jin-Xi WANG
}

\author{
Yulin University, No. 4, Chongwen Road, Yulin, Shaanxi, PR China, 719000 \\ mu_wjx001@163.com
}

Keywords: Dissolved Gas, Release Angle, Microbubble.

\begin{abstract}
The diameters of the microbubbles, which generate through gas-dissolving method, are determined by the factors such as gas components, structure of the releasers, dissolved gas pressures, and release angles, etc. The change tendencies of the diameters of the microbubbles with the dissolved gas pressures and release angles were studied through experiments, which were carried out by using a single component gas as nitrogen or oxygen. It is obtained from the experiment that the diameters of microbubbles increase with the increase in release angles, and decrease with the increase in dissolved gas pressures. The relationship between the diameters of the microbubbles and the influence factors were analyzed according to the experiment results. The study can provide some references for the using of single component gas-dissolving method to air floatation.
\end{abstract}

\section{Introduction}

Researches about the generation of bubbles are mainly focus on the bubble mechanics, such as the researches on the mechanical factors influencing the process of bubble floating under water[1], the change tendency of the microbubble diameters under the process of floating process[2], and the motion pattern of the microbubble under water[3]. However, fewer studies focus on the size of the microbubble and the factors influencing the size. The diameters of the microbubbles, which generate through gas-dissolving method, are determined by the factors such as gas components, structure of the releasers, dissolved gas pressures, and release angles, etc. The experiment method was adopted to study the change tendencies of the diameters of the microbubbles with the dissolved gas pressures and release angles. The microbubbles were generated through gas-dissolving method under pressure, the principle of which is based on the different solubility of gas in water under different pressures, the higher the pressure, the finer the bubbles and the more amount of the bubbles. In this study, two different single component gas, i.e. nitrogen and oxygen were adopted to study the diameter change tendency of microbubble under different release angles and dissolved gas pressures.

\section{Experimental Setup}

The experimental setup consists of water supply and air supply system, gas dissolving system (the fillable gas-dissolving tank), dissolved water release system and microbubble measuring and data acquisition system. An air compressor was used as air supply equipment in the experiment of gas dissolving and releasing, and nitrogen and oxygen were used in the experiments of nitrogen and oxygen gas dissolving and releasing, respectively.

Six different types of releasers with different release angles, which produce different pressures, were used, namely No. $1\left(20^{\circ}\right)$, No. $2\left(30^{\circ}\right)$, No. $3\left(45^{\circ}\right)$, No. $4\left(60^{\circ}\right)$, No. $5\left(90^{\circ}\right)$, and No. $6\left(180^{\circ}\right)$. The releasers are shown in Fig. 1.

The main purpose of the microbubble measuring and acquisition system is to capture the images of the bubble when leaving the releaser and rising slowly using a high-speed CCD camera with a microscope lens. Besides, other devices such as the LED backlight, the computer with gigabit network adapter, the "I" shaped glass sink, the microbubble device, the dissolved gas release device, the camera tripod, and the console are also needed. 


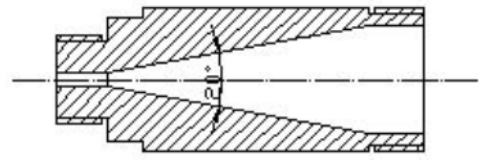

(a) Releaser No.1 (release angle $20^{\circ}$ )

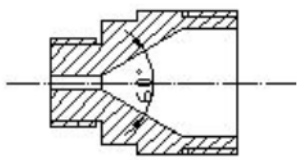

(d) Releaser No.4 (release angle $60^{\circ}$ )

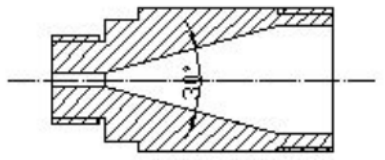

(b) Releaser No.2 (release angle $30^{\circ}$ )

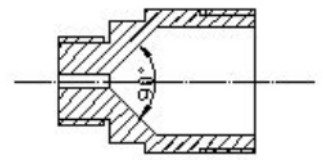

(e) Releaser No.5 (release angle $90^{\circ}$ )

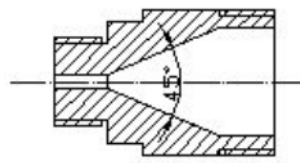

(c) Releaser No.3 (release angle $45^{\circ}$ )

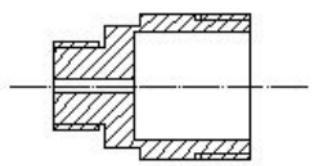

(f) Releaser No.6 (release angle $180^{\circ}$ )

Fig. 1 Different types of releaser

\section{Experimental Method}

The first step of the experiment is inject water to the dissolved gas tank and the sink. The injected water is $50 \%$ to $60 \%$ of tank's capacity [4], which can be determined by the level gauge. Then the valve of the pressure vessel with different gas is started, the gas is inflated into the tank with certain pressure. Before releasing the bubbles, the camera focus is calibrated by placing the transparent millimeter ruler into the center of the sink, and the camera cannot move after the calibration. After these preparation works, the release valve is turned on and the pictures are taken at the same time intervals. In this experiment, the opening of the valve is controlled by the flowmeter to ensure the flow rate is the same under different pressure.

\section{Results and Discussion}

\section{Effect of Release Angle on Bubble Diameter}

The relationship between the release angle and the average bubble diameter of nitrogen, oxygen, and air are shown in Fig. 2 (a), (b) and (c), respectively.

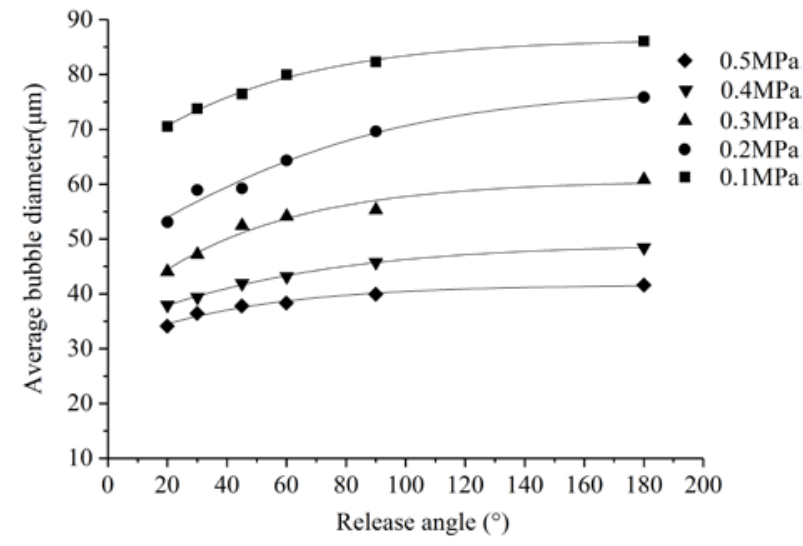

(a)Nitrogen

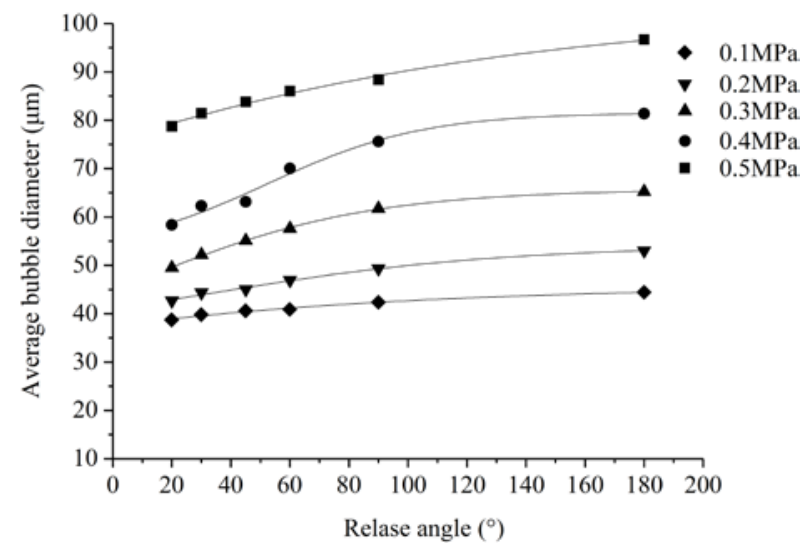

(b) Oxygen 


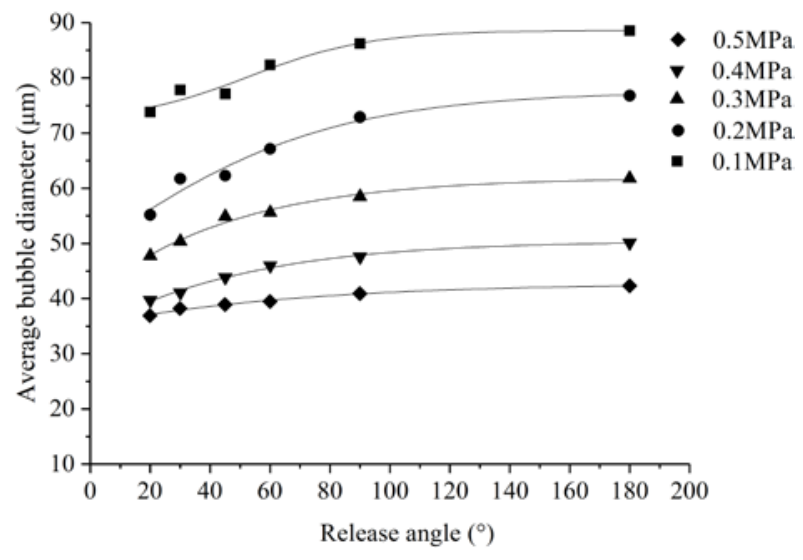

(c) Air

Fig. 2 The relationship between the release angle and the average bubble diameter

It can be known from Fig. 2 (a) to (c) that the average bubble diameter increases with the increase in release angle, and decreased with the increase in pressure of the dissolved gas. The larger the average bubble diameter, the better the floating capability, thus, small pressure dissolved gas is required.

\section{The Influence of Dissolved Gas Pressure on Average Bubble Diameter}

Three different gas, i.e. nitrogen, air, and oxygen, are adopted, and they are dissolved in 6 different releasers. The relationships between the pressure of the dissolved gases and the average bubble diameters are shown in

Fig. 3 (a) to (f).

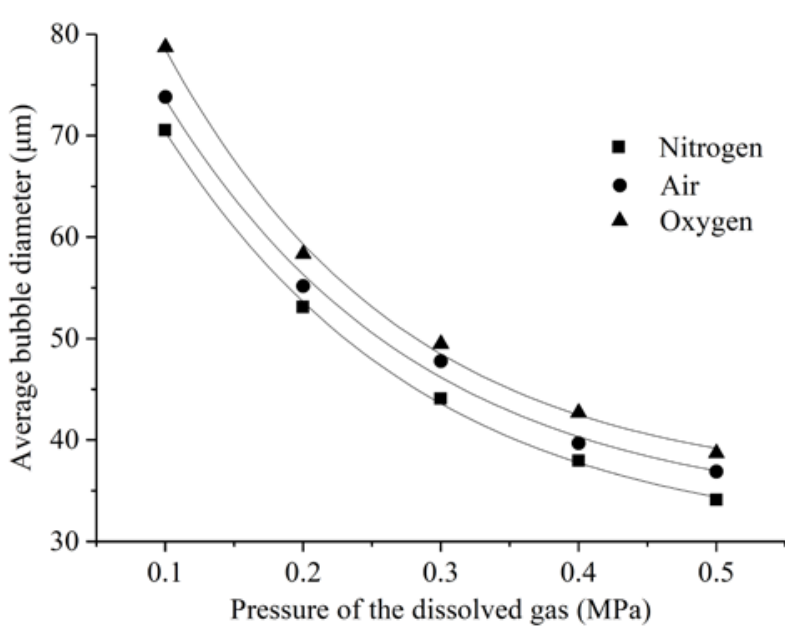

(a) Releaser No.1

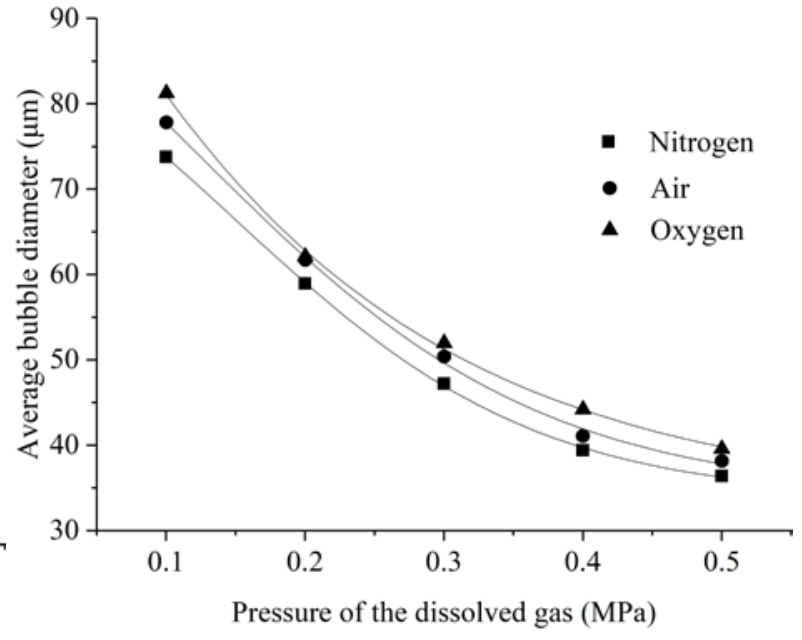

(b) Releaser No.2 


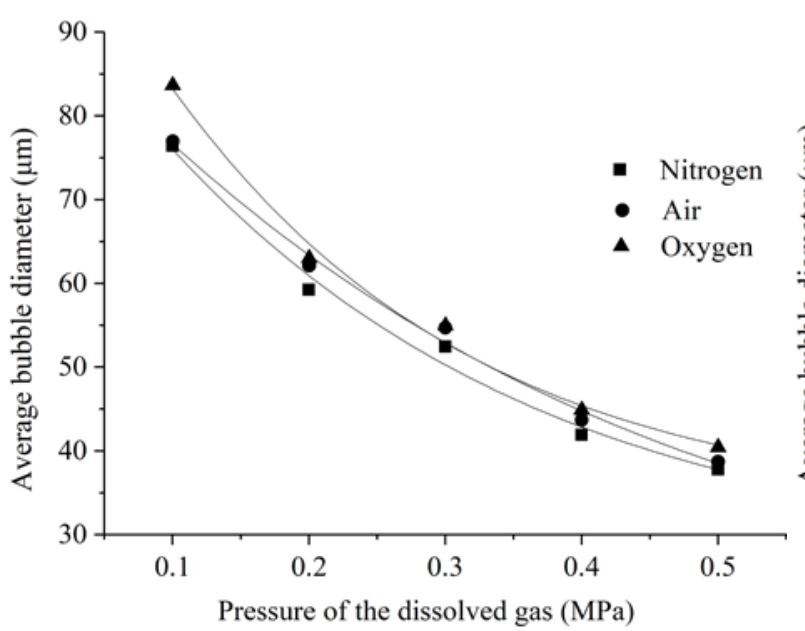

(c) Releaser No.3

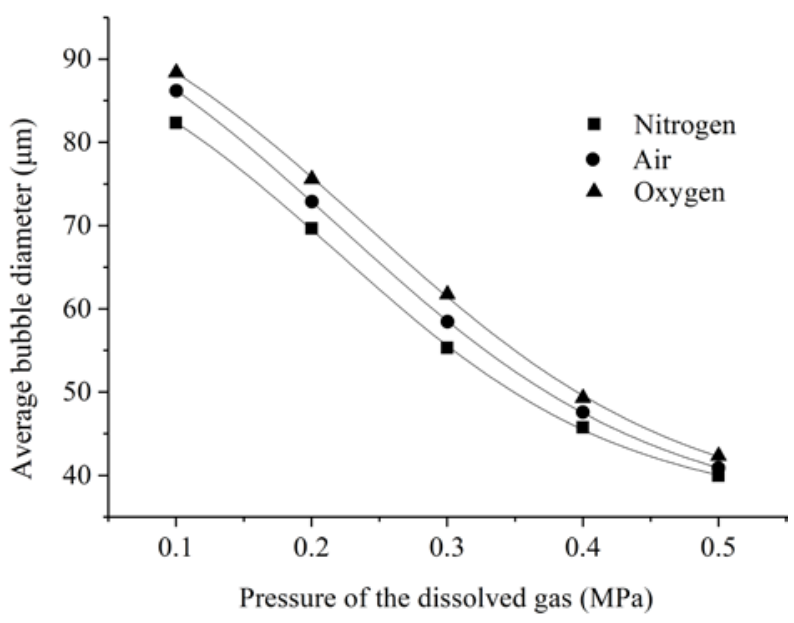

(e) Releaser No.5

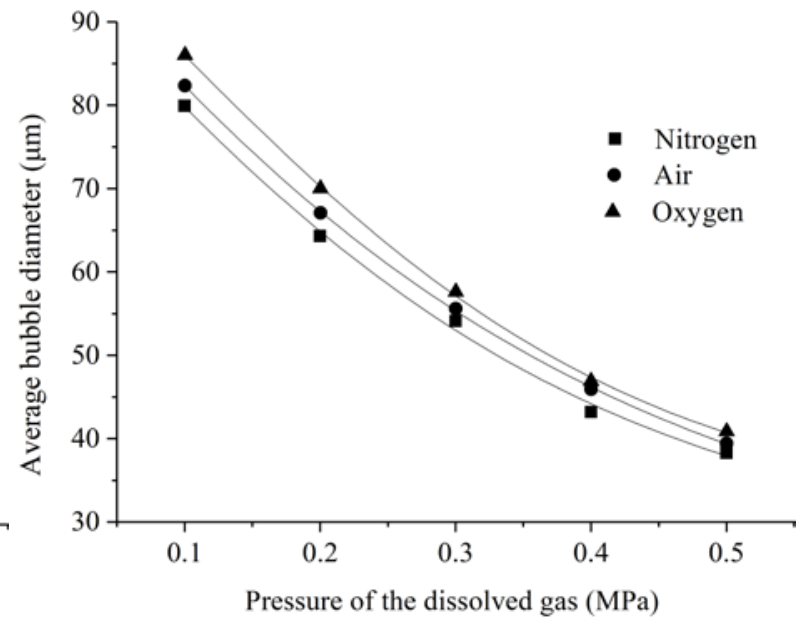

(d) Releaser No.4

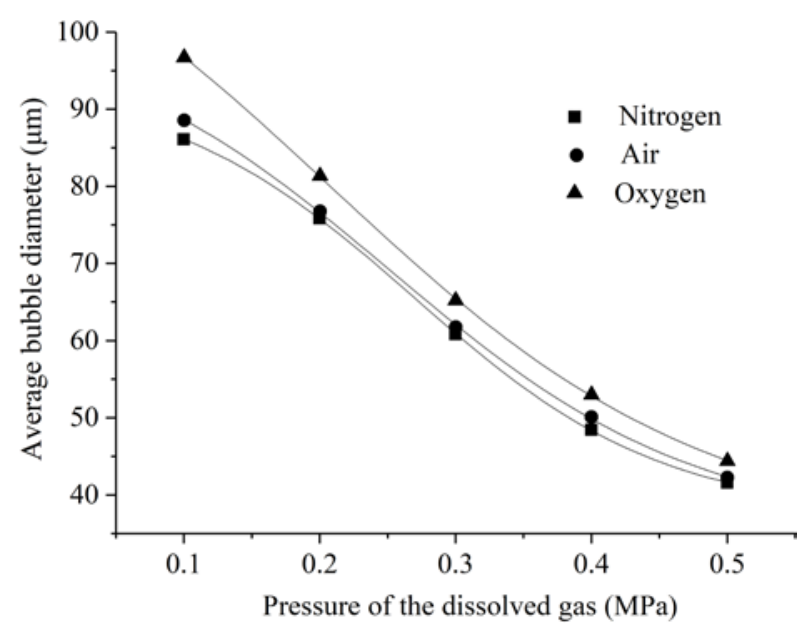

(f) Releaser No.6

Fig. 3 Average bubble diameter of 3 gases generated in different releasers

It can be seen from Fig. 3 that the trend of the average bubble diameters of the 3 different gases in different pressure are approximately the same. The average diameter of the bubbles generated from whatever nitrogen, air, or oxygen in the same releaser (i. e. the same release angle), all decrease with the increase in pressure of the dissolved gas. Thus, the pressure should be restrained to a small level. In addition, the average bubble diameters of the 3 gases in releaser No.6 are the largest in the 6 situations, which means that the larger the releaser angle, the larger the average bubble diameter. Moreover, the average bubble diameter of oxygen is the largest compared with the others, and the average bubble diameter of nitrogen is the smallest. Although nitrogen and oxygen molecules are non-polar, they have similar molecular structure and present the same form in water, but their solubility in water is different. The interaction between oxygen molecules and water molecules is stronger than the interaction between nitrogen molecules and water molecules. Therefore, the probability of collision in oxygen nucleation is greater than that in nitrogen. The collision makes more molecules aggregate together, which increases the average bubble diameter.

\section{Conclusions}

(1) The average bubble diameters of nitrogen, air, and oxygen are all increase with the increase in pressures of dissolved gas. In order to achieve a better flotation effect, the large average bubble diameter is needed, therefore, smaller pressure dissolved gas is required. However, the average bubble diameter almost not change if the pressure is greater than 0.4 . 
(2) The average bubble diameters of nitrogen, air, and oxygen are all increase with the increase in release angle.

\section{Acknowledgement}

This research was financially supported by Science and Technology Project of Yulin City (Gy13-01), Shaanxi Province, China.

\section{References}

[1] Shi Sheng-Wei, Wang Jiang-An, Jiang Xing-Zhou. Mechanics Effect Study of a Rising Micro-Bubble in Still Water [J]. Journal of Naval University of Engineering, 2008, 20(3): 83 87.

[2] Xu Mai-Rong, Liu Cheng-Yun. The Change of Radius and Velocity of the Rising Bubble in Water [J] College Physics, 2008, 27(11): 14 17.

[3] Liu Sheng, Yang Cheng-Yu, Wang Ping-Yi. Study on the Law of Bubble Movement in Water [J]. Journal of Chong Qing Jiaotong University, 2007, 26(3): 136 139-152.

[4] Wei Zai-Shan, Xu Xiao-Jun, Ning Ping, etc. Research and Advance in Wastewater Treatment by Flotation [J]. 2001, 1(4): 15-18. 